\title{
Canonical Ridge Analysis with Ridge Parameter Optimization
}

\author{
F. A.. Nielsen ${ }^{1}$, L. K. Hansen ${ }^{1}$, S. Strother ${ }^{2}$
}

${ }^{1}$ Technical University of Denmark, Lyngby, Denmark, ${ }^{2}$ VA Medical Center, PET Imaging

Service, Minneapolis, USA

\section{Introduction}

A number of linear multivariate statistical models have been applied for the analysis of functional images. We will here discuss a general model that encompasses several such approaches: Canonical Correlation Analysis (CCA), Partial Least Square (PLS), Orthonormalized Partial Least Square (OPLS), Ridge Regression and Fisher's Linear Discriminant (FLD). This general model is based on the concept of Canonical Ridge analysis [1]. The key matrix in this model is the "covariance" matrix:

$$
\mathbf{K}=\left(\left(1-k_{G}\right) \mathbf{G}^{\top} \mathbf{G}+k_{G} \mathbf{I}\right)^{-1 / 2} \mathbf{G}^{\top} \mathbf{X}\left(\left(1-k_{X}\right) \mathbf{X}^{\top} \mathbf{X}+k_{X} \mathbf{I}\right)^{-1 / 2}=\mathbf{U} \mathbf{\Lambda} \mathbf{V}^{\top}
$$

where the $\mathbf{X}$ is the datamatrix of size scans $\times$ voxels and $\mathbf{G}$ is a designmatrix of size scans $\times$ design variables, e.g., scans $\times$ effects or scans $\times$ groups. We are interested in the eigenvalues (singular values $\boldsymbol{\Lambda}$ ) and eigenvectors (eigenimages $\mathbf{V}$ and eigensequencies $\mathbf{U}$ ) of the $\mathbf{K}$ matrix. Setting $k_{X}=k_{G}=0$ leads to a matrix that has the same eigenvectors as those obtained through CCA. With $k_{X}=k_{G}=1$ the eigenvectors correspond to those of PLS and having $k_{X}=1$ and $k_{G}=0$ will yield the eigenvectors of the analysis Worsley has named Orthonormalized Partial Least Square [3]. The design variable should not be arbitrary scaled, either it should be orthonormalized or scaling should be estimated. The datamatrix will usually be degenerate: More voxels than scans. If the degenerate datamatrix is orthonormalized - as in CCA - the $\mathbf{K}$ matrix will have no structure in its "non-null" hyperspace it spans, i.e., its eigenvalues will be either one or zero. Such a problem does appear in PLS $\left(k_{X}=1\right)$. On the other hand, it does not provide the canonical vectors with the highest correlation as in CCA $\left(k_{X}=0\right)$ : We hypothesize that the optimal value of $k_{X}$ will be found in the interval ]0;1[ between the CCA and the PLS solution.

\section{Method}

We will optimize the $k_{X}$ parameter by comparing the first eigenimages (the first vectors in the two different V matrices). The $k_{G}$ we will fix at 0 , i.e., orthonormalize the designmatrix. The specific dataset we will use for illustration is a finger opposition experiment, single subject fMRI study consisting of 9 runs with 72 scans each. We split the dataset in two sets each comprising of 4 runs (the middle run discarded). For each set we use a "temporal" design matrix (72 groups - one group for each time point in the run [2]).

Result

We looked at the cross-correlation coefficient and the "distance from origin" measure from directional analysis [1] when comparing the two eigenimages. For the specific dataset we analyzed we found that the optimal $k_{X}$ parameter was neither 0 nor 1 , i.e., not the CCA or the PLS solution. The PLS decomposition (and Canonical Ridge decomposition with $k_{X}$ very close to 1 ) were dominated by noise, thus in one of the sets it was the second eigensequence that resembled the paradigm the most - not the first.

\section{Conclusion}

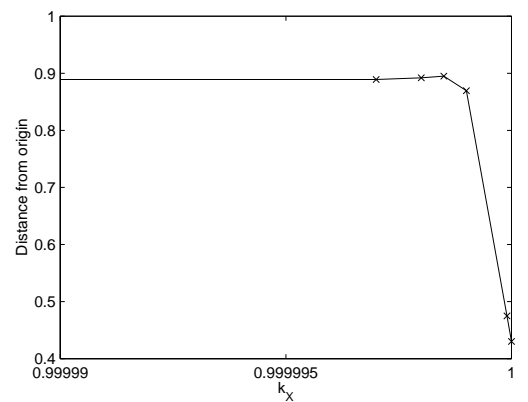

Our conclusion that is the Canonical Ridge analysis generalizes CCA and PLS, and that it is possible to optimize the Canonical Ridge parameter for reproducibility. Furthermore, that the eigenimage and eigensequence from the Canonical Ridge analysis are more generalizable than the corresponding eigenimages and eigensequences from both CCA and PLS.

Acknowledgment: This project has been funded by The Danish Research Councils and the Human Brain Project P20 MH57180 "Spatial and Temporal Patterns in Functional Neuroimaging".

\section{References}

1. Mardia, K. V., et al., Multivariate Analysis, Academic Press, 1979.

2. Strother, S. C., et al., 2nd Intl. Conf. on Functional Mapping of the Human Brain, 1996 S98.

3. Worsley, K., Human Brain Mapping, 1997, 5(4):254-258. 